\title{
A Corpus-Based Analysis of Synonyms-Take absolutely and thoroughly for example
}

\author{
JIN Ying-ying, QI Xiao-wen \\ University of Shanghai for Science and Technology, Shanghai, China
}

\begin{abstract}
Based on the British National Corpus (BNC), this paper makes an analysis of the word absolutely and thoroughly from their collocation. Therefore, the results can be listed as following. Firstly, the obvious adjective collocates of absolutely mainly are positive words while the obvious adjective collocates of thoroughly mainly are negative words. Secondly, the obvious verb collocates of absolutely mainly are negative words while the obvious verb collocates of thoroughly mainly are neutral words and negative words. This paper aims to make a comprehensive analysis of the synonyms, thus, contributing to foreign language learning and teaching for Chinese learners.
\end{abstract}

Keyword: absolutely, thoroughly, verb collocates, adjective collocates

\section{Introduction}

According to Lyons, synonyms are those words having similar meanings but not completely same. Thus, the analysis of synonyms is difficult but important for Chinese English learners. Traditionally, people often adopt the quantitative ways of introspection to analyze synonyms, depending on intuitions and experiences to give relevant prescriptions to the forms and usages of synonyms. Generally speaking, there are merely the meanings or part of sentences of the words in an English-Chinese dictionary, not related to the different usage of synonyms, which contributes to the misuse of synonyms for L2 learners. Therefore, corpus provides a new perspective for the analysis of synonyms, which makes complements for the traditional teaching ways. It makes an analysis in qualitative ways to show a more visualized, reliable, and convinced conclusion for us.

\section{Purpose}

The analysis of synonyms is important in language learning. Thus, this paper takes absolutely and thoroughly for example, making a conclusion of their MI score and collocation by concordance to analyze their difference and actual usages. On one hand, with the development of technology and society, corpus is extremely helpful for the analysis of synonyms. On the other hand, it’s really important for English teaching and learning.

\section{Object}

This paper takes absolutely and thoroughly (whose part of speech is adverbial) as the research object and makes a contrast between them.

JIN Ying-ying, M.A., University of Shanghai for Science and Technology, Shanghai, China.

QI Xiao-wen, Ph.D., associate professor, University of Shanghai for Science and Technology, Shanghai, China. 


\section{Methodology}

This paper makes use of the British National Corpus (BNC) (Web), which contains about one million words. It covers about the areas of spoken English, novels, magazines, newspapers, academic English, and so on. By means of BNC (Web), this paper makes a concordance of synonyms, then analyzes the index line to make a description, and finally makes a conclusion of their collocation.

\section{Results and Discussion}

The collocation of words is put forward by J. R. Firth, who is the representation of London School. In his opinion, the meanings of words can be reflected from their adjacent words. A collocation is a conventionally syntagmatic association of a string of lexical items which co-occur in a grammatical construct with mutual expectancy greater than chance as realization of non-idiomatic meaning in texts.

\section{The Adjective Collocation of the Word Absolutely and Thoroughly}

Before making a concordance of collocation in BNC, we need to set up some conditions as follows. Firstly, set the node as absolutely or thoroughly one by one. Then, set the part of speech of collocates as adjective [Adj.All] and input [j*]. Next, make the span as [0/+1]. Besides, make the sections as "IGNORE", the sorting conditions as "RELEVANCE" and the mutual information value (MI) > 3 (The MI score indicates the strength of a collocation and an MI score of 3 or higher can be taken to be significant. A high MI score often suggests that there is a high degree of co-occurrence in a particular text or a particular part of the corpus). Lastly, make the form of concordance as "Group by LEMMA". According to the above steps, this paper will make a concordance of collocates of absolutely and thoroughly twice. Then, this paper will make two tables to show the obvious adjective collocates of absolutely and thoroughly. The two tables both include word frequency, the word observed frequency, the percent of word frequency and MI score. Finally, this paper will choose 15 obvious adjectives as collocates of absolutely and thoroughly.

Table 1

The Obvious Adjective Collocation of Absolutely (Word Frequency $\geq 10$ )

\begin{tabular}{llllll}
\hline No. & Context & Frequency & All & $\%$ & MI \\
\hline 1 & [Disgusted] & 10 & 223 & 4.48 & 9.59 \\
2 & [Gorgeous] & 24 & 596 & 4.03 & 9.43 \\
3 & [Ludicrous] & 13 & 416 & 3.13 & 9.06 \\
4 & [Amazed] & 20 & 771 & 2.59 & 8.80 \\
5 & [Immaculate] & 10 & 456 & 2.19 & 8.55 \\
6 & [Delighted] & 47 & 2,181 & 2.15 & 8.53 \\
7 & [Fabulous] & 14 & 663 & 2.11 & 8.50 \\
8 & [Marvelous] & 35 & 1,746 & 2.00 & 8.42 \\
9 & [Fantastic] & 22 & 1,115 & 1.97 & 8.40 \\
10 & [Terrific] & 12 & 618 & 1.94 & 8.38 \\
11 & [Disgusting] & 14 & 719 & 1.89 & 8.14 \\
12 & [Brilliant] & 55 & 374 & 1.65 & 8.04 \\
13 & [Ridiculous] & 27 & 1,326 & 1.53 & 7.91 \\
14 & [Appalling] & 14 & 380 & 1.41 & 7.86 \\
15 & [Essential] & 121 & 320 & 1.36 & \\
\hline
\end{tabular}


From the above table, we can see that 15 adjectives can be classified into two types from the perspective of word-building: original adjective and derivative adjective. The former includes gorgeous, ludicrous, immaculate, fabulous, terrific, marvelous, fantastic, brilliant, ridiculous, and essential; the latter includes disgusted, amazed, delighted, disgusting, and appalling. From the perspective of semantics, they can be classified into two types: positive words and negative words. The positive words include gorgeous, immaculate, fabulous, terrific, marvelous, fantastic, brilliant, essential, and delighted; besides, by observing these positive words, we can find that all these positive words show a feeling of happiness of people or a kind of praise for someone or something. The negative words include ludicrous, ridiculous, disgusted, amazed, disgusting, and appalling. And all these negative words express negative feelings of human beings, including feeling sick of something and being foolish or shocked to do something.

Table 2

The Obvious Adjective Collocation of Thoroughly (Word Frequency $\geq 4$ )

\begin{tabular}{llllll}
\hline No. & Context & Frequency & All & $\%$ & MI \\
\hline 1 & [Enjoyable] & 21 & 893 & 2.35 & 10.12 \\
2 & [Soaked] & 4 & 213 & 1.88 & 9.79 \\
3 & [Unsatisfactory] & 10 & 753 & 1.33 & 9.29 \\
4 & [Bewildered] & 6 & 495 & 1.21 & 9.16 \\
5 & [Ashamed] & 8 & 1,016 & 0.79 & 8.54 \\
6 & [Unpleasant] & 9 & 1,235 & 0.73 & 8.43 \\
7 & [Annoyed] & 4 & 566 & 0.71 & 8.38 \\
8 & [Entertaining] & 4 & 828 & 0.48 & 7.83 \\
9 & [Familiar] & 22 & 5,531 & 0.40 & 7.55 \\
10 & [Charming] & 5 & 1,315 & 0.38 & 7.49 \\
11 & [Depressed] & 4 & 1,390 & 0.29 & 7.09 \\
12 & [Clean] & 25 & 8,817 & 0.28 & 7.07 \\
13 & [Nasty] & 4 & 1,855 & 0.22 & 6.67 \\
14 & [Upset] & 7 & 3,409 & 0.21 & 6.60 \\
15 & [Modern] & 20 & 12,869 & 0.16 & 6.20 \\
\hline
\end{tabular}

According to the above table, the obvious adjective can be classified into two types: adjective with negative affix and adjective without negative affix. The former includes unsatisfactory, ashamed, unpleasant, annoyed, and depressed. The latter includes enjoyable, soaked, bewildered, entertaining, familiar, charming, clean, nasty, upset, and modern. From the perspective of semantics, with the exception of enjoyable, entertaining, familiar, charming, clean, and modern, the rest, for example, soaked, unsatisfactory, bewildered, ashamed, unpleasant, annoyed, depressed, nasty, and upset, all are negative words. That is to say, these negative words mainly show the feelings of unhappiness or unsatisfactory, which is embodied a kind of critic attitude towards something.

Therefore, from all the above, we can make a conclusion that the obvious adjective collocations of absolutely are mainly positive words, which show a feeling of happiness or a kind of praise for someone or something. While the obvious adjective collocations of thoroughly are mainly negative words, which shows the feelings of unhappiness or unsatisfactory. Besides, original adjectives and derivative adjectives can be collocated with the word absolutely. 


\section{The Verb Collocation of the Word Absolutely and Thoroughly}

Before making a concordance of collocation in BNC, we need to set up some conditions as follows. Firstly, set the node as absolutely and thoroughly one by one. Then, set the part of speech of collocates as verb [Verb.All] and input [ $\left.\mathrm{v}^{*}\right]$. Next, make the span as $[-1 /+1]$. Besides, make the sections as "IGNORE", the sorting conditions as "RELEVANCE" and the mutual information value (MI) > 3(MI is used to measure the degree of collocation of different words). Lastly, make the form of concordance as "Group by LEMMA". According to the above steps, this paper will make a concordance of collocates of absolutely and thoroughly twice. Then, this paper will make two tables to show the obvious verb collocates of absolutely and thoroughly. The two tables both include word frequency, the observed frequency, the percent of word frequency and MI score. Finally, this paper will choose 15 obvious verbs as collocates of absolutely and thoroughly.

Table 3

The Obvious Verb Collocates of Absolutely (Word Frequency $\geq 5$ )

\begin{tabular}{llllll}
\hline No. & Context & Frequency & All & $\%$ & MI \\
\hline 1 & [Devastate] & 17 & 576 & 2.95 & 7.98 \\
2 & [Terrify] & 21 & 888 & 2.35 & 7.66 \\
3 & [Horrify] & 10 & 482 & 2.07 & 7.47 \\
4 & [Adore] & 9 & 492 & 1.83 & 7.29 \\
5 & [Stun] & 9 & 578 & 1.56 & 6.06 \\
6 & [Shatter] & 13 & 876 & 1.48 & 6.66 \\
7 & [Thrill] & 12 & 1,016 & 1.18 & 6.65 \\
8 & [Forbid] & 15 & 1,280 & 1.17 & 6.04 \\
9 & [Exhausted] & 12 & 1,567 & 0.77 & 4.86 \\
10 & [Stagger] & 6 & 887 & 0,68 & 4.38 \\
11 & [Refuse] & 32 & 10,458 & 0.31 & 4.15 \\
12 & [Convince] & 7 & 2,886 & 0.24 & 4.10 \\
13 & [Delight] & 6 & 2,886 & 0.21 & 3.99 \\
14 & [Hate] & 10 & 4,998 & 0.20 & 0.19 \\
\hline
\end{tabular}

From the above table, we can make an analysis from the perspective of semantics. All the above collocates can be divided into two types: positive words and negative words. The former includes adore, convince, delight, and guarantee. The latter includes devastate, terrify, horrify, stun, shatter, thrill, forbid, exhaust, stagger, refuse, and hate. Therefore, the obvious verb collocates of absolutely mainly are negative words. And all these negative words.

Table 4

The Obvious Verb Collocates of Thoroughly (Word Frequency $\geq 4$ )

\begin{tabular}{llllll}
\hline No. & Context & Frequency & All & $\%$ & MI \\
\hline 1 & [Imbue] & 4 & 176 & 2.27 & 10.07 \\
2 & [Saturate] & 4 & 193 & 2.07 & 9.94 \\
3 & [Disapprove] & 9 & 442 & 2.04 & 9.91 \\
4 & [Enjoy] & 147 & 14,046 & 1.05 & 8.95 \\
5 & [Deserve] & 24 & 2,970 & 0.81 & 8.58 \\
6 & [Confuse] & 13 & 2,082 & 0.62 & 8.20 \\
7 & [Spoil] & 8 & 1,372 & 0.58 & 8.11 \\
\hline
\end{tabular}




\begin{tabular}{llllll}
\multicolumn{2}{l}{ (table 4 continued) } \\
\hline No. & Context & Frequency & All & $\%$ & MI \\
\hline 8 & [Investigate] & 28 & 5,332 & 0.53 & 7.96 \\
9 & [Recommend] & 17 & 5,827 & 0.29 & 7.11 \\
10 & [Mix] & 14 & 5,531 & 0.25 & 6.90 \\
11 & [Alarm] & 7 & 3,054 & 0.23 & 6.76 \\
12 & [Upset] & 7 & 3,409 & 0.21 & 6.60 \\
13 & [Explore] & 9 & 4,737 & 0.19 & 6.49 \\
14 & [Integrate] & 4 & 2,169 & 0.18 & 6.45 \\
15 & [Wash] & 9 & 5,522 & 0.16 & 6.27 \\
\hline
\end{tabular}

From the above table, we can make an analysis from the perspective of semantics. All the above collocates of thoroughly can be divided into three types: positive words, neutral words, and negative words. The positive words include enjoy and deserve. The neutral words include investigate, mix, explore, recommend, wash, and integrate. The negative words include imbue, saturate, disapprove, confuse, spoil, alarm, and upset. So the obvious verb collocates of thoroughly mainly are neutral words and negative words. However, the above words can hardly distinguish them clearly from the perspective of pragmatic purpose. The characters of these words are not obvious.

Therefore, we can make a conclusion that the obvious verb collocates of absolutely mainly are negative words, which reveals that people feel terrible or afraid to do something or something is disastrous. While the obvious verb collocates of thoroughly mainly are neutral words and negative words, which is not obvious in their characters.

\section{Conclusion}

Based on the BNC, this paper makes an analysis of the word absolutely and thoroughly from their collocation. Therefore, three aspects can be listed as following. Firstly, the obvious adjective collocations of absolutely are mainly positive words, which show a feeling of happiness or a kind of praise for someone or something. While the obvious adjective collocations of thoroughly are mainly negative words, which shows the feelings of unhappiness or unsatisfactory. Secondly, the obvious verb collocates of absolutely mainly are negative words, which reveal that people feel terrible or afraid to do something or something is disastrous. While the obvious verb collocates of thoroughly mainly are neutral words and negative words, which are not obvious in their characters. Therefore, all the above conclusions are the results of the analysis of absolutely and thoroughly.

\section{References}

Wei, N. X. (2002a). A corpus-based and corpus-driven analysis of collocation [J]. Contemporary Linguistics, 4 (2), 101-114.

Wei, N. X. (2002b). Conventional methods of semantic prosody study [J]. Foreign Language Teaching and Research, 34(4), 300-307.

Yang, C. X. (2014). A corpus-based analysis of synonyms-Take suspect and doubt for example [J]. Journal of Southwest University of Science and Technology, 31(5), 45-49. 